\title{
Allergisches Kontaktekzem auf Metronidazol
}

\author{
V. Prugovečki \\ D. Mechtel \\ B. Knopf
}

\section{Allergic Contact Dermatitis Due to Metronidazole}

\section{Zusammenfassung}

Topisch verwendetes Metronidazol gehört zur Standardtherapie der Rosazea. Die Behandlung gilt als sicher und nebenwirkungsarm. Wir berichten über den äußerst seltenen Fall eines allergischen Kontaktekzems nach Anwendung von Metronidazol bei einer 45-jährigen Frau. Nach durchgeführtem Epikutantest und repetitivem offenen Anwendungstest (ROAT) mit Metronidazol, die positiv ausfielen, kam es zu einer Verschlechterung des klinischen Bildes mit Körperstreuherden.

\section{Abstract}

Topical metronidazole is known as a standard therapy of rosacea. Treatment is persumed to be safe and to rarely cause side effects. We report a rare case of a 45 -year-old woman with contact dermatitis due to metronidazole. After positive patch testing and repeated open application test (ROAT) the dermatitis became worse and spread over her body.

\section{Einleitung}

Die Rosazea ist eine chronische Hauterkrankung mit zentrofazialen Erythemen, Papeln, Pusteln sowie Bindegewebs- und Talgdrüsenhyperplasien. Metronidazolhaltige Externa zählen heutzutage insbesondere in der Behandlung der Rosacea papulopustulosa zu den Standardtherapeutika, da sie eine sehr gute Wirkung haben und kaum Nebenwirkungen verursachen. Wir stellen mit dieser Kasuistik den seltenen Fall eines allergischen Kontaktekzems auf Metronidazol vor.

\section{Fallbericht}

\section{Anamnese}

Die sonst gesunde 45-jährige Patientin berichtete zwei Jahre vor der jetzigen stationären Aufnahme erstmalig wegen einer Rosazea mit einem erythromycin- und metronidazolhaltigen Exter- num behandelt worden zu sein. Hierunter kam es zunächst zu einer Befundbesserung. Mehrere Wochen bevor wir die Patientin erstmalig sahen sei es zu einer akuten Verschlechterung der Hautveränderungen gekommen, weshalb ihr erneut eine $2 \%$ ige metronidazolhaltige Creme sowie Minocyclin Filmtabletten $50 \mathrm{mg}$ (Minakne ${ }^{\circledR}$ ) verordnet wurden, wovon die Patientin insgesamt fünf Tabletten einnahm. Wegen zunehmender Befundverschlechterung wurde nach drei Behandlungstagen die Lokaltherapie auf Prednicarbat Salbe (Dermatop ${ }^{\circledR}$ ), den Gerbstoff Tannosynt ${ }^{\circledR}$ Lotio und Ungt. Metronidazoli SR umgestellt und die Patientin erhielt Methylprednisolon Tabletten (Urbason ${ }^{\circledR}$ ) $42 \mathrm{mg}$ für einen Tag, $32 \mathrm{mg}$ für einen weiteren Tag und jeweils $16 \mathrm{mg}$ an den beiden nächsten Tagen. Da sich der Hautbefund weiter verschlimmerte, erfolgte die Einweisung der Patientin in unsere Klinik. 


\section{Hautbefund}

Bei der Aufnahme bestand ein ausgeprägtes Spannungs- und Hitzegefühl im Gesicht. Die Haut war düsterrot verfärbt, infiltriert, z.T. nässend und mit gelben Krusten belegt (Abb. 1).

\section{Diagnostik \\ Laboruntersuchungen}

Bis auf eine Eosinophilie von 9,1\% (Normalwert bis 6,0\%) bei einer mit 4,7 109/1 im Normbereich liegender Leukozytenzahl und einen Harnstoffwert von $3,2 \mathrm{mmol} / \mathrm{l}$ (Normalwert 3,6 - 7,1 mmol/l) fanden sich unauffällige Werte für BSG, Blutbild, Differenzialblutbild, klinische Chemie, Elektrophorese, TSH, Blutzucker, ANF-Titer, ds-DNS-Titer, Urinstatus und -sediment. Das Gesamt-IgE betrug 40,2 IU/ml.

\section{Mikrobiologie}

Im Wangenabstrich konnte Staphylococcus epidermidis nachgewiesen werden. Die mykologische Untersuchung (Abstrich und Kultur) fiel negativ aus.

\section{Therapie und Verlauf}

Wir begannen eine Therapie mit Bolus-alba-Masken, Schwarzteeumschlägen und Dermatop ${ }^{\circledR}$ Basisfettsalbe sowie Doxycyclin 100-mg-Tabletten $2 \times$ täglich. Unter dieser Therapie kam es zu einer deutlichen Befundbesserung mit Abklingen des ausgeprägten Erythems und Ödems des Gesichtes sowie gleichzeitigem Nachlassen des subjektiven Spannungsgefühls. Nach 14 Tagen führten wir eine Epikutantestung und einen Anwendungstest mit Metronidazol durch.

\section{Allergologische Hauttestungen Epikutan-Test}

Auf dem erscheinungsfreien Rücken der Patientin wurden mittels Finn Chambers on Scanpor ${ }^{\circledR}$ die Allergene der Reihen Standard, Salbengrundlagen und Emulgatoren und Kosmetik/Haushalt (Fa. Hermal) sowie Metronidazol 0,75\% in Vaseline getestet. Nach 24, 48 und 72 Stunden kam es zu einfach positiven Testreaktionen auf Dibromdicyanobutan/Phenoxyethanol $(1: 4)$ und Metronidazol (Abb. 2).

\section{ROAT}

Ein in der Ellenbeuge des linken Armes durchgeführter repeated open application test (ROAT) mit Metronidazol Gel 0,75\% war bereits nach 24 Stunden positiv (Abb. 3).

Das Metronidazol-Gel wurde von unserer Apotheke hergestellt und hatte folgende Zusammensetzung: Metronidazol 1,5 g, Propylenglykol $10 \mathrm{~g}$, Natriumetidat $0,2 \mathrm{~g}$, Tramethanol $0,5 \mathrm{~g}$, Polyacrylsäure 1,0 g, Kaliumsorbat 0,2 g, Wasser ad $200 \mathrm{~g}$.

Unter der Testung kam es zu einer akuten Verschlechterung des Hautbefundes mit Streureaktionen an Stamm und Extremitäten. Unter Fortführung der therapeutischen Maßnahmen trat schrittweise Besserung ein.

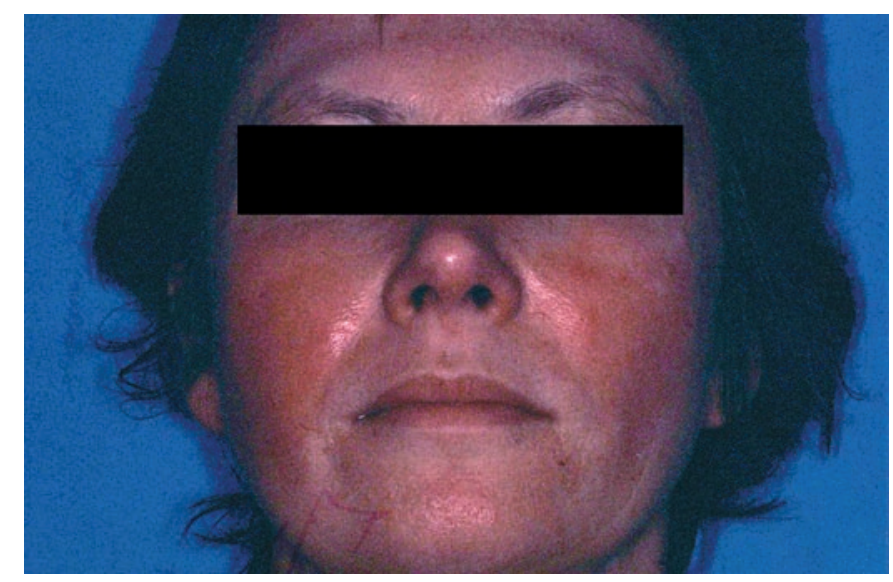

Abb. 1 Klinischer Befund des Gesichts bei erstmaliger Vorstellung.

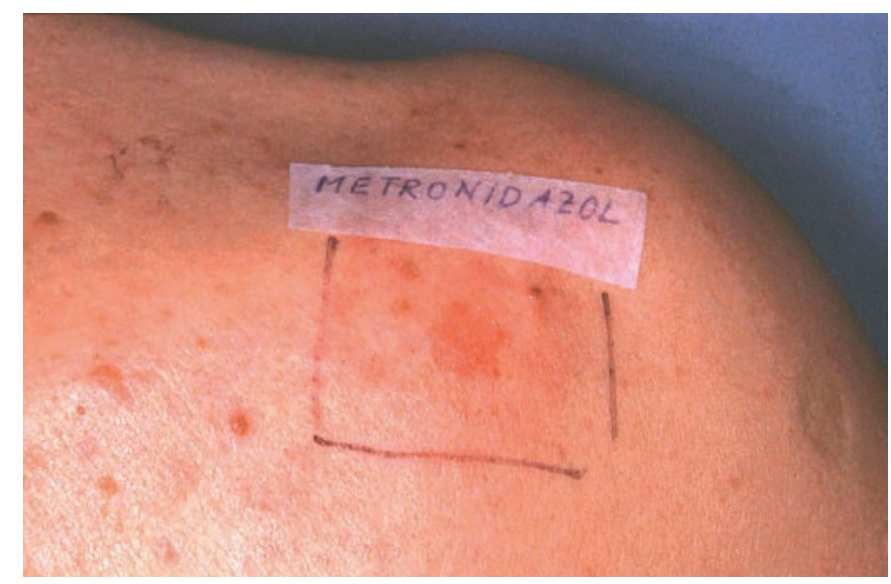

Abb. 2 Positives Epikutantestergebnis auf Metronidazol.

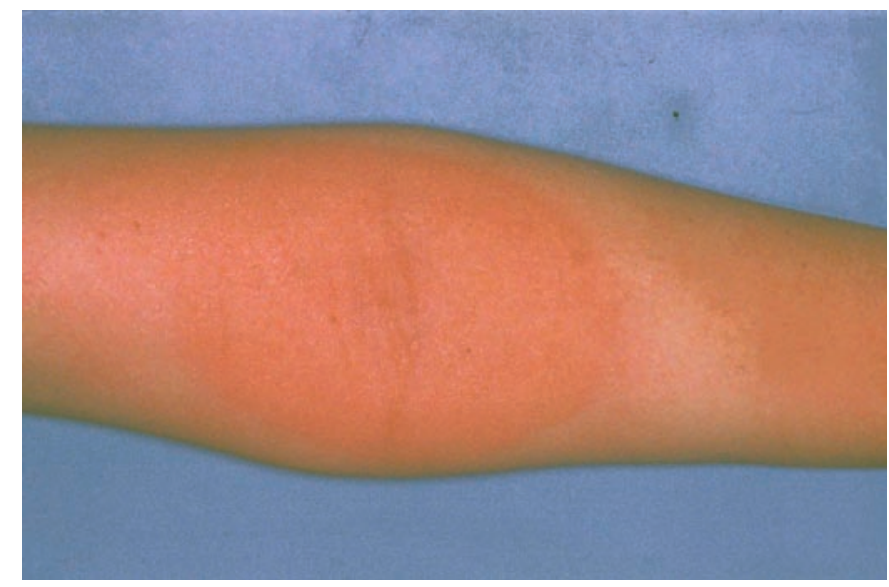

Abb. 3 Positiver ROAT (repeated open application test) auf Metronidazol.

\section{Diskussion}

Metronidazol $\left(\mathrm{C}_{6} \mathrm{H}_{9} \mathrm{~N}_{3} \mathrm{O}_{3}\right)$, auch 2-(2-Methyl-5-nitro-1-imidazolyl)-ethanol, mit einem Molekulargewicht von 171,16 und einem Schmelzpunkt bei $158-160^{\circ} \mathrm{C}$ ist ein Nitroimidazol-Derivat. Metronidazol hat eine Halbwertszeit von 7-8 Stunden bzw. seine Metaboliten von 10 Stunden. Der Hauptmetabolit von Metro- 
nidazol ist das Hydroxymetronidazol. Nach lokaler Applikation ist Metronidazol im Serum entweder gar nicht oder nur in sehr geringen Mengen nachweisbar [1]. Es kommt zur intrazellulären Metabolisierung unter anaeroben Bedingungen und Anlagerung der Metaboliten an die DNA.

Metronidazol fängt freie Elektronen ab und verringert so die Entstehung reaktiver Sauerstoffspezies (ROS), die Stoffwechselveränderungen des Elastins und Kollagens bedingen. Metronidazol wirkt antientzündlich sowie proliferationshemmend und immunmodulierend. Der genaue Wirkmechanismus, durch welchen Metronidazol die entzündlichen Veränderungen bei der Rosazea vermindert, ist jedoch immer noch nicht vollständig aufgeklärt.

Zur Behandlung der Rosacea werden im Allgemeinen 0,75-, 1oder $2 \%$ ige Präparationen in Form von Cremes, Gelen oder Lotionen 1-2 × täglich dünn aufgetragen.

In dem Fertigarzneimittel Metrogel ${ }^{\circledR}$ liegt Metronidazol in einem Polyacrylat-Wassergel vor. Da sich Metronidazol in Wasser schlecht löst, wird der Wirkstoff aus dem Wasser-Gel schneller freigesetzt. Fetthaltige Creme- oder Salben-Rezepturen binden den Wirkstoff dagegen stärker. Der hohe Wassergehalt der GelFormulierung steigert somit die lokale Penetration und Bioverfügbarkeit [2].

Lokal appliziertes Metronidazol wird im Allgemeinen sehr gut vertragen. Allergische Reaktionen auf Metronidazol sind äußerst selten.

Feldman u. Mitarb. [3] stellten dar, dass entsprechend einer zwischen den Jahren 1990 und 1997 in den USA durchgeführten Datenerhebung an über 1,1 Millionen Patienten zur topischen Behandlung der Rosazea am häufigsten Metronidazol verschrieben wurde. In der Arbeit wird auf die hohe Sicherheit der Behandlung mit topischen metronidazolhaltigen Präparationen hingewiesen.

Shelanski u. Mitarb. [4] untersuchten in einer Epikutanteststudie die Sicherheit bestimmter dermatologischer Externa bei 108 gesunden Erwachsenen und fanden unter anderem, dass Metronidazol-Gel kein klinisch signifikantes Irritations- oder Sensibilisierungspotenzial besitzt.

Metronidazol wird wegen seiner antibakteriellen und antiprotozoalen Aktivität auch häufig zur peroralen Anwendung verordnet. Es gibt wenige Berichte über Unverträglichkeitsreaktionen durch oral eingenommenes Metronidazol. Short u. Mitarb. [5] berichteten über einen Patienten mit einem durch Metronidazol hervorgerufenen fixen Arzneimittelexanthem, das durch Epikutantestung in loco reproduzierbar war.

Walfish und Sapadin [6] beschreiben eine Kreuzsensitivität auf Doxycyclin und Metronidazol, wobei es sich hierbei lediglich um assoziierte Sensibilisierungen handeln könnte.

Es gibt bisher wenige Veröffentlichungen über allergische Kontaktekzeme durch topisch angewandtes Metronidazol.
1992 berichteten Izu u. Mitarb. [7] über eine 30-jährige Frau mit einer Kontaktdermatitis auf Thioconazol (ein Phenylethylimidazol) mit positiven Testreaktionen und somit wahrscheinlicher Kreuzsensitivität auf Bifonazol (ein Phenylmethylimidazol) und Metronidazol (ein Nitroimidazol).

Im Jahre 1997 beschrieben Vincenzi u. Mitarb. [8] den Fall einer 26-jährigen Frau mit einer Kontaktdermatitis im Gesicht nach Applikation eines 1\%igen metronidazolhaltigen Gels. Im Epikutantest fanden sich am Tag 2 und 3 positive Reaktionen auf das Metronidazol, wobei es zu keiner positiven Testreaktion auf die anderen getesteten Imidazolderivate kam.

Hjerppe u. Mitarb. [9] berichteten im Jahre 2000 über eine 55-jährige Frau mit einem Kontaktekzem auf Metronidazol bei positiven Epikutantestergebnissen.

Choudry u. Mitarb. [10] veröffentlichten 2002 den Fall einer 59-jährigen Frau mit einer Rosazea, die Metrogel ${ }^{\circledR}$ angewandt hatte. Im Epikutantest konnte eine 2fach positive Reaktion auf 2-Bromo-2-Nitropropan,1,3-diol (Bronopol), ein Konservierungsmittel in Metrogel ${ }^{\circledR}$, am Tag 4 nachgewiesen werden.

Bei der von uns vorgestellten Patientin entwickelte sich im Rahmen der ambulanten Behandlung ihrer Rosazea mit 2\%igem Metronidazol eine Sensibilisierung gegenüber diesem Arzneimittel. Bei erneuter lokaler Anwendung von Metronidazol 0,75\% kam es zur Ausbildung eines akuten allergischen Kontaktekzems. Die Epikutantestung von Metronidazol inklusive ROAT führten nicht nur zu positiven Testreaktionen, sondern auch zu ausgeprägten hämatogenen Streuherden. Einer von uns geplanten Testung auf weitere Inhalts- und Konservierungsstoffe des Fertigpräparates Metrogel $^{\circledR}$ sowie auf weitere Imidazole zur Feststellung einer möglichen Kreuzreaktivität stand die Patientin ablehnend gegenüber.

Eine Sensibilisierung gegenüber Benzoaten, die als Methyl4-hydroxybenzoat und Propyl-4-hydroxybenzoat in Metrogel ${ }^{\circledR}$ enthalten sind, ist jedoch unwahrscheinlich, da die Patientin im Epikutantest gegenüber Natriumbenzoat in der Reihe Kosmetik/ Haushalt nicht reagierte.

Zwischen der bei der Patientin zusätzlich bestehenden Sensibilisierung gegenüber dem Konservierungsstoff Dibromdicyanobu$\tan /$ Phenoxyethanol, das häufig in einem Verhältnis von $1: 4$ als Euxyl ${ }^{\circledR} \mathrm{K} 400$ in unterschiedlichen Haut- und Körperpflegemitteln aber auch im technischen Bereich (z. B. als Kühlschmiermittel) vorkommen kann, und der bestehenden Metronidazol-Gel Unverträglichkeit besteht kein ursächlicher Zusammenhang, da es in der Metronidazol-Präparation nicht als Konservierungsstoff enthalten ist. Möglicherweise handelt es sich um eine so genannte stumme Sensibilisierung gegenüber Dibromdicyanobu$\tan /$ Phenoxyethanol, wahrscheinlich durch die Anwendung von Kosmetika.

Resümierend können wir feststellen, dass die topische Anwendung von Metronidazol in der Behandlung der Rosazea zur Standardtherapie gehört. Unverträglichkeiten sind nur selten zu beobachten. Das Sensibilisierungspotenzial von Imidazolderivaten ist als eher schwach einzuschätzen. Die bei der Therapie mit 
Metronidazol-Externa auftretenden häufigsten Nebenwirkungen wie Brennen, Erythem und Pruritus klingen nach einer initialen Phase meist schnell ab und führen bei entsprechender Aufklärung der Patienten in seltenen Fällen zu Therapieabbrüchen. Kommt es unter der Behandlung mit Metronidazol-Externa allerdings zu keiner Besserung oder sogar Verschlechterung des Hautbefundes, so muss auch an das Vorliegen eines Kontaktekzems auf Metronidazol gedacht und eine allergologische Hauttestung durchgeführt werden.

Nach unserem Wissen ist dies der erste im deutschsprachigen Raum veröffentlichte Fall eines allergischen Kontaktekzems auf Metronidazol.
Literatur

${ }^{1}$ McClellan KJ, Noble S. Topical Metronidazole. A Review of its Use in Rosacea. Am J Clin Dermatol 2000; 1 (3): 191 - 199

2 Metrogel ${ }^{\circledR}$ Kurzmonografie. Galderma Deutschland

${ }^{3}$ Feldman SR, Hollar CB, Gupta AK, Fleischer AB Jr. Woman commonly seek care for rosacea: dermatologists frequently provide the care. $\mathrm{Cu}-$ tis 2001; 68: $156-160$

${ }^{4}$ Shelanski MS, Phillips SB, Potts CE. Evaluation of cutaneous reactivity to recently marketed dermatologic products. Int J Dermatol 1996; 35: $137-140$

${ }^{5}$ Short KA, Fuller LC, Salisbury JR. Fixed drug eruption following metronidazole therapy and the use of topical provocation testing in diagnosis. Clin Exp Dermatol 2002; 27: 464 - 466

${ }^{6}$ Walfish AE, Sapadin AN. Fixed drug eruption due to doxycycline and metronidazole. Cutis 2002; 69: 207-208

${ }^{7}$ Izu R, Aguirre A, González M, Díaz-Pérez JL. Contact dermatitis from tioconazole with cross-sensitivity to other imidazoles. Contact Dermatitis 1992; 26: 130-131

${ }^{8}$ Vincenzi C, Lucente P, Ricci C, Tosti A. Facial contact dermatitis due to metronidazole. Contact Dermatitis 1997; 36: 116-117

${ }^{9}$ Hjerppe M, Karppinen A, Hasan T. Metrdonidatsolivoiteesta allerginen ihottuma. Duodecim 2000; 116: 2229-2231

${ }^{10}$ Choudry K, Beck MH, Muston HL. Allergic contact dermatitis from 2-bromo-2-nitropropane-1,3-diol in Metrogel ${ }^{\circledR}$. Contact Dermatitis 2002; 46: $60-61$

\section{Preise}

Paul Caesar Richter-Preis für Geschichte der Dermatologie und Venerologie

Auf dem DDG-Kongress 2005 in Dresden wird die Arbeitsgemeinschaft für Geschichte der Dermatologie und Venerologie (AGDV) e.V. die beste Arbeit zum Thema Geschichte der Dermatologie und Venerologie auszeichnen.

Zum ersten Mal wurde der Paul Caesar Richter-Preis ausgeschrieben. Dieser Preis ist nach einen Dermatologen benannt, der sich als einer der ersten intensiv mit der Fachgeschichte befasst hat. Paul Richter lebte von 1865 - 1938 in Berlin, war niedergelassener Dermatologe, und erwarb sich als Medizinhistoriker bleibenden Ruf.

Ausgezeichnet werden Arbeiten, welche die Wichtigkeit der Beschäftigung mit geschichtlichen Fragen in der Dermatologie für die heutige klinische Tätigkeit unterstreichen und richtungsweisend für die Auseinandersetzung mit der Fachgeschichte sind. Akzeptiert werden unveröffentlichte Manuskripte und Arbeiten, die seit 2002 publiziert wurden. Der maximale Umfang sollte 20 DIN A4-Seiten nicht übersteigen. Bewerber können ihre in deutscher Sprache verfassten Arbeiten bis zum 15. Februar 2005 einreichen.

Die Bewertung wird von einem vom Vorstand bestellten Kuratorium unter Ausschluss des Rechtsweges vorgenommen. Das Preisgeld in Höhe von $1000 €$ wird von der Firma Stiefel Laboratorium GmbH, Offenbach, die sich bereits in der Vergangenheit für die Belange der Medizingeschichte eingesetzt hat, gesponsort. Die Preisverleihung findet während der 44. Tagung der Deutschen Dermatologischen Gesellschaft in Dresden statt. Weitere Informationen unter: www.agdv.org
Preis der Sächsischen Gesellschaft für Dermatologie e.V. für die Nachwuchsförderung in Sachsen erstmalig verliehen

Anlässlich der 5. Gemeinschaftstagung des Berufsverbandes der Deutschen Dermatologen e.V., des Landesverbandes Sachsen und der Sächsischen Gesellschaft für Dermatologie e.V. vom 1. - 3. Oktober 2004 in Oberwiesenthal wurde erstmalig der Preis für Nachwuchsförderung der Sächsischen Gesellschaft für Dermatologie e.V. verliehen, der von der Firma Bioglan Pharma $\mathrm{GmbH}$ (Greifswald) gesponsert wurde.

Das Preiskomitee unter Leitung von Herrn Prof. Dr. B. Knopf (Zwickau) hatte sich die Aufgabe nicht leicht gemacht. Unter den Einsendungen wurde die Arbeit „Das Schicksal der jüdischen Dermatologen Deutschlands zur Zeit des Nationalsozialismus“ von Herrn Dr. med. Sven Eppinger (TU Dresden; Abb.1) ausgewählt und mit einem Preisgeld von $1000 €$ prämiert. Gewürdigt wurde insbesondere die kritische Auseinandersetzung mit der Wissenschaftsgeschichte des eigenen Fachgebietes.

Nachwuchsförderung und Nachwuchsgewinnung sind zwei untrennbare Seiten der regionalen Arbeit. Die Sächsische Gesellschaft für Dermatologie e.V. sieht diese Aufgabe als Herausforderung für die Gestaltung der Zukunft in Sachsen an.

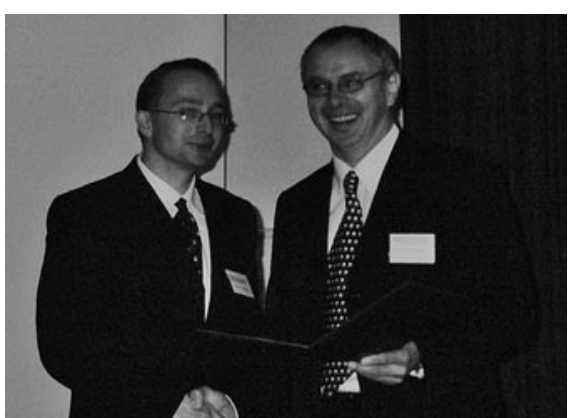

Abb. 1 Verleihung des ersten Nachwuchspreises der Sächsischen Dermatologischen Gesellschaft an Herrn Dr. S. Eppinger (links) durch den Vorsitzenden der Gesellschaft, Herrn Prof. Dr. U. Wollina. 